\title{
Interactive comment on "Radiance-based Retrieval Bias Mitigation for the MOPITT Instrument: The Version 8 Product” by Merritt N. Deeter et al.
}

\section{Merritt N. Deeter et al.}

mnd@ucar.edu

Received and published: 24 May 2019

See attached pdf file.

Please also note the supplement to this comment:

https://www.atmos-meas-tech-discuss.net/amt-2019-41/amt-2019-41-AC2-

supplement.pdf 